\title{
A Systematic Review and Meta-Analysis for Better Measurement of Listening Effort in Adults with Hearing Loss
}

\author{
Chanbeom Kwak ${ }^{1,2}$, Woojae Han ${ }^{1,2,3}$ \\ ${ }^{1}$ Laboratory of Hearing and Technology, ${ }^{2}$ Division of Speech Pathology and Audiology, ${ }^{3}$ Research Institute of Audiology and Speech Pathology, \\ College of Natural Sciences, Hallym University, Chuncheon, Korea
}

\author{
Received: September 17, 2020 \\ Revised: October 8, 2020 \\ Accepted: October 8, 2020 \\ Correspondence: \\ Woojae Han, PhD \\ Division of Speech Pathology \\ and Audiology, \\ 8603 Natural Science Building, \\ Hallym University, 1 Hallymdaehak-gil, \\ Chuncheon 24252, Korea \\ Tel: +82-33-248-2216 \\ Fax: +82-33-256-3420 \\ E-mail: woojaehan@hallym.ac.kr
}

\begin{abstract}
The present study aimed to identify better tools for measuring listening effort in the hearing-impaired and/or hearing aid users and to suggest its clinical implication by using systematic review and meta-analysis. To search articles from six electronic databases, 'listening effort', 'hearing loss/hearing impaired', and 'hearing aid(s)' were used as their key terms. Although 8,761 articles were found initially, only 19 articles which met the inclusion criteria were applied to the further review and analysis. In a checklist of the study quality, there was no significant difference between the articles. Based on meta-analysis of three subgroups (i.e., objective, subjective, and self-report rating), the objective measurements showed statistically significant and the highest effect size $(1.031,95 \%$ confidence interval: $-0.106-2.169)$, whereas the self-report rating had the lowest effect size $(-1.280,95 \%$ confidence interval: $-4.180-1.620)$ with no significance. Although the funnel plot was asymmetrical, the Egger's regression asymmetry test revealed no publication bias. In sum, the objective measurement showed the most effective way to evaluate the listening effort for the hearing-impaired and/or hearing aid users. However, there was a non-negligible variance between the types of measurements. In the following study, we suggest to investigate certain relationship between listening effort and speech perception performance in the hearing-impaired and/or hearing aid users and to establish standardized criteria for clinical purposes of the listening effort.
\end{abstract}

Key Words: Hearing aid, Hearing loss, Listening effort, Objective measure, Self-report rating, Subjective measure.

\section{INTRODUCTION}

청취 노력(listening effort)은 청각적으로 제시되는 자극에 대한 이해와 이를 기반으로 과제를 수행함에 있어 요구되는 인 지적 과정이며(McGarrigle et al., 2014), 과제 수행에 따른 부정 적인 영향을 최소화하기 위해 의도적이고 자발적으로 발현되 는 인지적 자원을 의미한다(Pichora-Fuller et al., 2016). 실제 로 청취 노력은 과제를 수행하는데 사용 가능한 수단으로 정의 될 수 있는 인지적 자원(cognitive resources), 동기부여(motivation), 노력(effort) 등을 포함한 다차원적 인지 과정의 결과로 발현되기 때문에(Pichora-Fuller et al., 2016), 이를 측정함에 있어서 인지적 자원의 발현 여부, 발현 과정에 대한 세부적 이 해, 가시적으로 노력 정도를 측정할 수 있는 데이터 도출 등 다

(c) This is an Open Access article distributed under the terms of the Creative Commons Attribution Non-Commercial License (https://creativecommons.org/licenses/by-nc/4.0) which permits unrestricted non-commercial use, distribution, and reproduction in any medium, provided the original work is properly cited.
양한 관점에서 연구들이 선행되었다.

1973년 Kahneman에 의해 제안된 인지 능력 모델(cognitive capacity model)에 따르면, 주의력과 노력은 전체 능력에 국한 되며, 동시 작업을 수행하는 사람의 능력은 작업에 필요한 요구 정도에 따라 달라진다. 즉, 과제를 수행함에 있어서 발현되는 인 지적 자원은 유한하며 이는 과제의 난이도가 상승함에 따라 인 지적 자원의 사용 역시 증가함을 나타낸다(Kahneman, 1973). 이러한 주장은 수년 전부터 심리-청각-인지 분야에 적용되어 청취 노력의 메커니즘 및 효과적인 측정 방법을 제시하고자 하 였다. 청각적 관점에서 제한된 인지 능력 모델을 재해석하면, 청 력 손실이나 큰 배경 소음으로 인해 청취가 어려운 상황에서는 어음을 인지하기 위해 더 많은 집중과 노력이 요구되게 된다. 이때, 집중과 노력을 위해 상당량의 인지적 자원이 요구되고 어 음의 정확한 문맥적 이해를 위해 인지적 자원이 과하게 사용되 기 때문에 전체 인지적 자원 중 어음 정보를 해석하고 저장하 기 위한 잔존 자원이 부족하게 되며(Pichora-Fuller et al., 
2016; Wingfield et al., 2006) 궁극적으로 청자는 그러한 청취 상황에서 쉽게 피로하거나 집중을 포기하게 된다.

여러 연구들에서 이미 밝혔지만, 난청인들은 배경 소음이나 반향음 등 청취에 어려운 환경에서 저조한 어음인지를 보이며 (Ayasse et al., 2017; Ayasse \& Wingfield, 2018; Desjardins \& Doherty, 2013; Huber et al., 2018; Krueger et al., 2017; Picou \& Ricketts, 2018; Ricketts et al., 2019; Ward et al., 2017; Zekveld et al., 2009), 보청기나 인공와우 등과 같은 청각보조기기 를 활용하여 청력 역치의 보완은 물론 저조한 어음인지도의 향 상을 기대한다(Alhanbali et al., 2018; Alhanbali et al., 2019; Desjardins \& Doherty, 2013; Giroud et al., 2017; Krueger et al., 2017; Mackersie et al., 2015; Picou \& Ricketts, 2018; Ricketts et al., 2019; Zekveld et al., 2009). 최근 학계에서는 이러한 난청인들의 어음인지력 향상을 위해 요구되는 인지적 자 원인 청취 노력을 재해석하고 있다. Pichora-Fuller et al.(2016) 은 Framework for Understanding Effortful Listening (FUEL) 을 설명하면서 기존 연구들은 보청기 착용을 통한 난청인들의 가청력 보완 및 더 나은 어음인지력의 향상이 주요한 목적이었 으나, 이를 향상시키기 위해 오히려 난청인들에게 요구되는 과 도한 청취 노력은 바람직하지 않다고 주장하였다. 난청 노인을 대상으로 객관적 측정 방법인 동공 확장을 사용하여 언어적 복잡성에 따른 문장 인지와 청취 노력에 대해 보고한 Ayasse \& Wingfield(2018)의 연구에서도 난청 노인은 건청 노인에 비 해 검사의 난이도가 어려워짐에 따라 동공의 확장이 유의미하 게 발생하였다. 다시 말해, 동일한 언어 능력을 보이더라도 난청 으로 인한 문장 인지의 저조한 수행력과 더불어 과제의 복잡성 으로 유발되는 과도한 인지적 자원은 난청인에게 더 높은 청취 노력을 야기하게 되는 것으로 설명할 수 있다. 그러나 높은 어 음인지력을 수행하기 위해 수반되는 과도한 인지적 노력으로 인해 난청인들은 쉽게 피로감을 느끼게 되고 어려운 청취 환경 을 기피하는 등 장기적 관점에서는 난청인의 수행력에 부정적 인 영향을 미치게 된다. 어음인지도와 자가 보고로 측정한 청 취 노력 간 연관성에 대해 연구한 Krueger et al.(2017)의 결과 역시, 배경 소음의 정도가 증가함에 따라 장노년층 난청 그룹의 어음인지도가 건청 성인의 그룹에 비해 급격하게 감소할 뿐만 아니라, 이에 요구되는 청취 노력 역시 난청 그룹이 유의미하게 더 높았다. 또한, 연구에 참여한 보청기 착용자인 난청인들에게 보청기 착용을 통해 일차적으로 어음인지도를 향상시켰더라도, 보청기가 청력과 인지 기능의 복합적인 과정으로 발현되는 청 취 노력을 완화/감소시키기에는 한계가 있음을 시사하였다. 선 행연구 결과들을 종합해보면, 난청인은 건청인에 비해 동일한 청취 상황에서 요구되는 청취 노력의 정도가 더 높으며 보청기 를 통한 가청력과 어음인지도의 향상으로도 과도하게 요구되
는 청취 노력을 감소시키는 것에는 한계가 있음을 보였다.

현재 청취 노력의 측정 방법에 있어서는 표준화된 검사 도구 와 효과적인 측정 방법에 대한 검증의 부재로 인해 연구별로 측정 방법이 다소 상이하다. Kahneman(1973)의 연구에서 보 고한 제한된 인지 자원 이론에 근거한 FUEL (Pichora-Fuller et al., 2016)의 모형 역시 청취 노력을 측정하는 방법에 대해서 인지 행동적 반응(dual-task paradigm, 회상 검사, 반응/응답 시간), 뇌파 반응(electroencephalography, $\mathrm{EEG}$ ), 자율신경계 반응(동공 확장, 피부 전도성, 심박수), 자가 보고(Likert-scale) 의 측정 방법에 따른 반응을 분류하였으나 현재까지 청취 노력 의 임상적 적용을 위해 검증된 측정 도구의 부재를 한계점으로 언급한 바 있다. 따라서 본 논문에서는 체계적인 문헌 고찰과 메타 분석을 활용하여 난청인 및 보청기 착용자를 대상으로 기 존에 연구된 청취 노력의 측정 도구 및 방법에 대해 효과를 확 인하고 심도 있게 논의하고자 한다. 더불어 향후 클리닉 혹은 청각 센터 등에서 청취 노력의 측정 및 해석의 상용화를 위해 임상적 적용에 대해 제언하고자 한다.

\section{MATERIALS AND METHODS}

\section{Search strategy}

체계적인 문헌 고찰과 메타 분석을 위해 Moher et al.(2015) 의 Preferred Reporting Items for Systematic Reviews and Meta-analysis Protocols (PRISMA-P)와 Centre for Reviews and Dissemination(2009)의 Participants, Intervention, Control, Outcomes, Study design (PICOS) 기준에 근거하여 문헌 의 조사, 선별, 선정, 분석을 시행하였다. 구체적으로, 문헌의 조 사, 선별, 및 선정에 대한 과정은 PRISMA-P에 근거한 흐름도 를 사용하여 나타내었다(Figure 1). 더불어, 문헌의 선별은 $\mathrm{Ta}-$ ble 1 에 작성한 PICOS 기준을 적용하여 본 연구에서 설정한 목적과 일치하지 않는 문헌들을 배제하였다.

\section{Article selection}

문헌의 조사, 선별, 선정을 위해 Embase, Medline, Pubmed, Web of Science, Science Direct, Cumulative Index to Nursing and Allied Health의 총 6개의 전자 데이터베이스 검색 엔 진에 대해 2002년 1월 1일에서 2020년 5월 1일까지 출간된 문 헌 중 '청취 노력(listening effort)', '난청(hearing loss/hearing impaired)', '보청기(hearing aid/hearing aids)'의 검색어를 포 함하는 문헌들을 모두 조사하였다.

전자 데이터베이스의 검색 결과, 총 9,715 편의 문헌이 검색되 었다(identification process) (Figure 1). 954편의 중복 문헌을 제거한 후 8,761 편의 문헌에 대해 제시된 초록을 기준으로 선별 


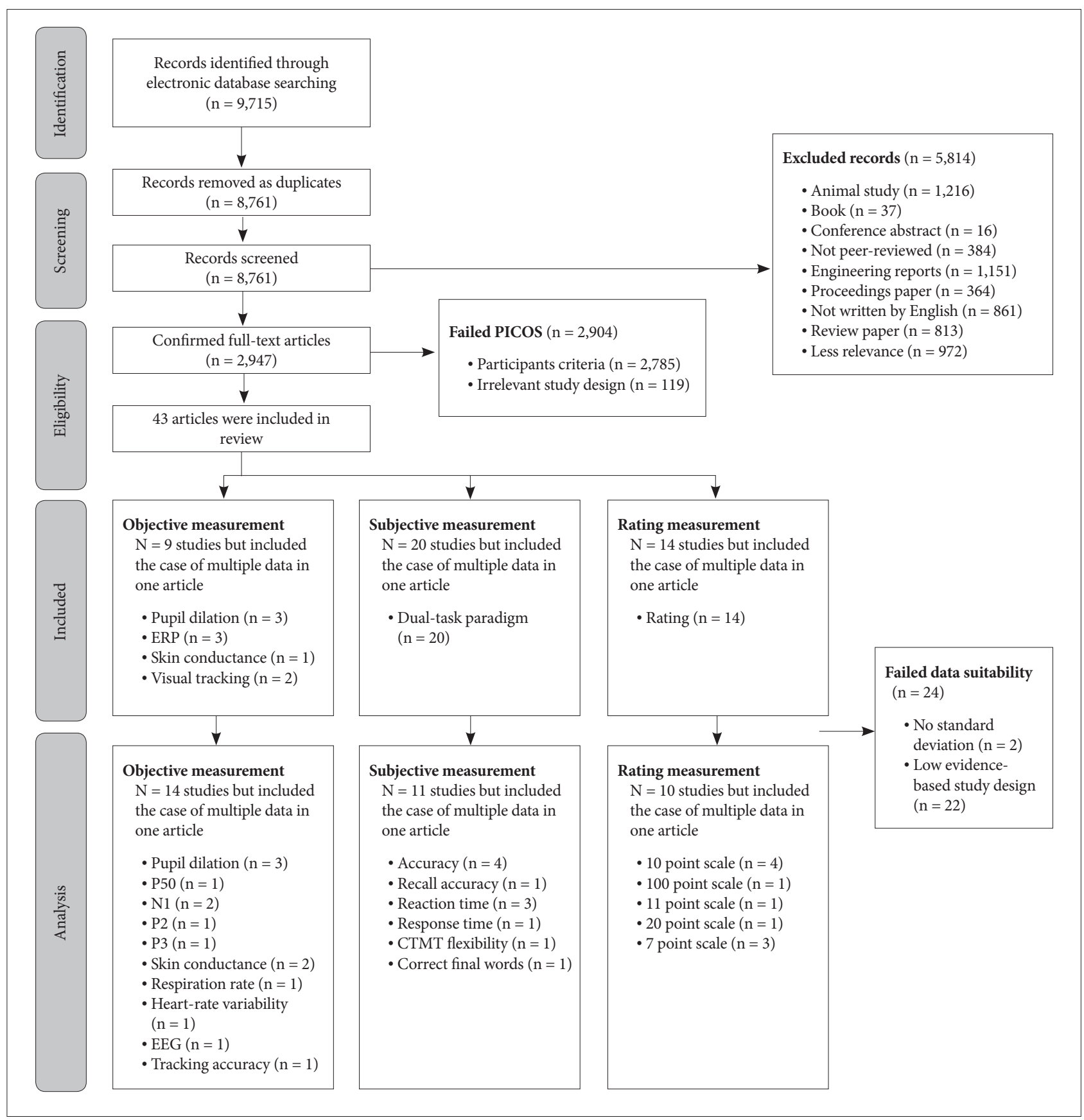

Figure 1. Flowchart of the study search and selection process based on PICOS criteria. PICOS: Participants, Intervention, Control, Outcomes, Study design, ERP: event-related potential, EEG: electroencephalography, CTMT: Comprehensive Trail-Making Test.

Table 1. Inclusion criteria based on the Participants, Intervention, Control, Outcomes, and Study designs (PICOS) strategy

\begin{tabular}{ll}
\hline \multicolumn{1}{c}{ PICOS } & \multicolumn{1}{c}{ Contents } \\
\hline Participants & Adults (18+ years) with hearing loss and/or hearing aid \\
Intervention & Objective, subjective, and self-report rating measurements \\
Control & Adults (18+ years) with normal hearing or repeated measures \\
Outcomes & Listening effort expressed by objective (i.e., pupil dilation), subjective, (i.e., accuracy), and rating scale (i.e., Likert scale) \\
Study designs & $\begin{array}{c}\text { Randomized controlled trials, non-randomized controlled trials, cohort studies, between-group comparison, and } \\
\text { repeated measures with additional purposes }\end{array}$ \\
\hline
\end{tabular}


과정을 진행하였다. 선별 과정에서 총 5,814 편의 문헌이 배제 기준에 해당되어 제외되었고(screening process) (Figure 1), 문 헌 선별 과정을 통과한 2,947편의 문헌에 대해서는 PICOS 기 준에 근거하여 전체 본문의 내용을 검토하였다. 이후 Table 1 의 $\mathrm{PICOS}$ 기준을 충족하지 못한 총 2,904편의 문헌이 제외되었다 (eligibility process) (Figure 1). 모든 기준을 충족한 최종 43편 이 본 연구의 문헌의 질적 평가, 데이터 추출 및 분석에 포함되 었으며 청취 노력의 측정 방법에 따른 효과 크기를 추정하기 위해 하위군 분석으로 객관적 측정(objective measurement), 주관적 측정(subjective measurement), 자가 보고 측정(selfreport rating)의 3가지 측정 방법에 따라 문헌을 분류하였다 (included process) (Figure 1). 그러나 문헌별 데이터 추출 과정 에서 표준 편차 데이터 누락(2편) 및 낮은 근거 기반 연구 설계 (22편)을 보인 24편의 문헌이 추가적으로 배제되었다. 즉, 낮은 근거 기반 연구 설계는 PICOS 기준에 근거하여 무작위 대조 실험(randomized controlled trial) 이 아닌 그룹 내 비교 분석 (one-group comparison) 연구 설계를 대상으로 하였다.

총 19 편의 문헌에서 하위군 분석 기준에 따라 데이터 추출을 시행하였으며, 동일 문헌에서 다중 측정 방법의 시행 및 결과를 포함하여 총 35건이 메타 분석에 포함되었다(analysis process)
(Figure 1).

\section{Study quality and potential sources of study bias}

문헌의 질적 평가와 연구의 잠재적 편향은 CAMARADES checklist (PROSPERO, 2014)를 기준으로 6개 항목에 대해 구 체적으로 평가하였다. 예를 들어, 본 연구에 포함된 총 19편의 개별 문헌을 대상으로 무작위 추출(randomization), 대조군 (controls), 표본 수 산정(sample size calculation), 맹검 출판 여부(publication after peer review), 결과 측정(outcome measure), 잠재적 이해관계 충돌(statement of potential conflict of interest)의 항목을 바탕으로 질적 평가를 수행하였다(Table 2).

각 항목별 점수는 ‘예’ 혹은 ‘아니요의 이분형 문항으로 측정 하여 각각 1점과 0점을 부여하였다. 부여된 항목별 점수는 Grading of Recommendations, Assessment, Development, and Evaluation (GRADE) Working Group(2004)의 가이드라 인에 근거하여 각 문헌의 질적 수준을 평가하였다. 즉, 측정한 전체 점수가 높으면 해당 문헌은 연구의 질적 수준이 높음을 의미한다.

Table 2. Scientific study validity criteria based on CAMARADES checklists (PROSPERO, 2014)

\begin{tabular}{|c|c|c|c|c|c|c|c|}
\hline \multirow[b]{2}{*}{ Article } & \multicolumn{6}{|c|}{ Scientific study validity criteria } & \multirow[b]{2}{*}{$\begin{array}{c}\text { Study quality } \\
\text { score }\end{array}$} \\
\hline & Randomization & Controls & $\begin{array}{l}\text { Sample size } \\
\text { calculation }\end{array}$ & $\begin{array}{c}\text { Publication } \\
\text { after peer } \\
\text { review }\end{array}$ & $\begin{array}{l}\text { Outcome } \\
\text { measure }\end{array}$ & $\begin{array}{c}\text { Statement } \\
\text { of potential } \\
\text { conflict } \\
\text { of interest }\end{array}$ & \\
\hline Ayasse \& Wingfield, 2018 & 0 & 1 & 0 & 1 & 1 & 1 & 4 \\
\hline Desjardins \& Doherty, 2013 & 0 & 1 & 1 & 1 & 1 & 1 & 5 \\
\hline Ward et al., 2017 & 0 & 1 & 0 & 1 & 1 & 1 & 4 \\
\hline Tun et al., 2009 & 0 & 1 & 0 & 1 & 1 & 0 & 3 \\
\hline Ayasse et al., 2017 & 0 & 1 & 0 & 1 & 1 & 1 & 4 \\
\hline Rosemann \& Thiel, 2020 & 0 & 1 & 0 & 1 & 1 & 1 & 4 \\
\hline Bertoli \& Bodmer, 2014 & 1 & 1 & 0 & 1 & 1 & 0 & 4 \\
\hline Giroud et al., 2017 & 1 & 1 & 0 & 1 & 1 & 0 & 4 \\
\hline Bernarding et al., 2013 & 1 & 1 & 0 & 1 & 1 & 0 & 4 \\
\hline Zekveld et al., 2009 & 1 & 1 & 0 & 1 & 1 & 0 & 4 \\
\hline Rosemann \& Thiel, 2019 & 0 & 1 & 0 & 1 & 1 & 0 & 3 \\
\hline Mackersie et al., 2015 & 1 & 1 & 1 & 1 & 1 & 0 & 5 \\
\hline Alhanbali et al., 2019 & 1 & 1 & 0 & 1 & 1 & 1 & 5 \\
\hline Krueger et al., 2017 & 1 & 1 & 0 & 1 & 1 & 0 & 4 \\
\hline Holube et al., 2016 & 1 & 1 & 0 & 1 & 1 & 1 & 5 \\
\hline Picou \& Ricketts, 2018 & 1 & 0 & 0 & 1 & 1 & 1 & 4 \\
\hline Huber et al., 2018 & 1 & 1 & 0 & 1 & 1 & 1 & 5 \\
\hline Ricketts et al., 2019 & 1 & 0 & 0 & 1 & 1 & 1 & 4 \\
\hline Alhanbali et al., 2018 & 0 & 0 & 1 & 1 & 1 & 1 & 4 \\
\hline
\end{tabular}

1 and 0 refer to "Yes" and "No", respectively 


\section{Meta-analysis}

본 연구에 포함된 총 35 편의 문헌에서 추출 및 합성한 데이 터가 메타 분석에 적합 여부를 확인 후, Comprehensive MetaAnalysis (Ver. 3, Biostat Inc., Englewood, NJ, USA)를 사용 하여 분석을 실시하였다. 메타 분석은 추출 및 합성된 데이터 의 특성에 따라 효과 크기(effect size)를 계산하는데 사용되는 측도가 결정된다(Kang, 2015). 본 연구에 포함된 문헌 35건의 데이터 중 20건은 실험군과 대조군의 평균과 표준편차를, 나머 지 15 건은 상관관계 결과를 보고하였기에 각 문헌의 평균, 표 준편차, 상관관계 결과를 바탕으로 청취 노력에 대한 효과 크 기를 추정하였다.

메타 분석의 특성 상 대다수의 문헌들이 청취 노력을 측정하 기 위해 사용한 측정 방법이 상이하기 때문에, 표준화된 평균 차이(standardized mean difference, SMD)를 적용하여 효과 크기를 추정하고 변량 효과 모형의 $95 \%$ 의 신뢰구간(confidence interval, CI)에서 검증하였다. 깔때기 그림(funnel plot) 의 산점도와 Egger's regression asymmetry test의 유의 확률 $(p<0.05)$ 을 통해 출판 편향을 확인하였다. 또한 Cochrane's $\mathrm{Q}$ test와 Higgins $\mathrm{I}^{2}$ statistics를 근거로 각 문헌 간 분산의 이질 성(heterogeneity)를 확인하였다. Cochrane's Q test는 $95 \%$ 의 유의수준 $(p<0.05)$ 을 기준으로 이질성의 여부를 판단하며, Higgin's $I^{2}$ statistics는 $0 \%$ 에서 $100 \%$ 의 값으로 이질성에 대한 양적 기준을 제시한다. $\mathrm{I}^{2}$ 이 $0 \%$ 에서 $25 \%$ 는 낮은 수준의 이질 성, $25 \%$ 에서 $75 \%$ 는 중간 수준의 이질성, $75 \%$ 에서 $100 \%$ 는 높 은 수준의 이질성을 의미한다(Higgins et al., 2003).

\section{RESULTS}

\section{Scores of study quality}

CAMARADES checklist (PROSPERO, 2014)를 기준으로 평가한 문헌의 질적 평가와 연구의 잠재적 편향은 통계/데이터마 이닝 및 그래프를 위한 언어인 R 프로그램(R Core Team, 2018) 을 사용하여 카이 제곱 검정(chi-square test)을 수행하였다.

질적 평가에 대한 전체 문헌들의 평균은 4.20 (standard deviation: 0.62 , range: 3 5)이었고, 문헌의 질적 평가에 대한 적 합도 확인을 위한 카이 제곱 검정 결과는 문헌의 질적 평가 간 유의미한 차이를 보이지 않았다 $\left(\chi^{2}=3.7536, \mathrm{df}=19, p>0.05\right)$.

\section{Characteristic analysis of studies in meta-analysis}

Table 3은 PICOS 기준에 따라 각 문헌의 결과를 요약하여 정리하였다. 대상자는 난청 혹은 보청기를 착용한 18 세 이상의 성인이었으며, 19편의 문헌에는 난청을 가진 노인(Ayasse et al., 2017; Ayasse \& Wingfield 2018; Huber at al., 2018; Tun et al.,
2009), 보청기를 착용한 노인(Giroud et al., 2017), 난청 노인과 보 청기 착용 노인(Alhanbali et al., 2018), 난청을 가진 장년(Bernarding et al., 2013), 난청 장년과 보청기 착용 장년(Zekveld et al., 2009), 난청을 가진 장년과 노인(Bertoli \& Bodmer, 2014; Holube et al., 2016; Rosemann \& Thiel, 2019; Rosemann \& Thiel, 2020; Ward et al., 2017), 보청기를 착용한 장년과 노인 (Desjardins \& Doherty, 2013; Krueger et al., 2017; Ricketts et al., 2019), 난청 장년 및 노인과 보청기 착용 장년 및 노인 (Alhanbali et al., 2019; Picou \& Ricketts, 2018), 난청 청장년 및 노인과 보청기 착용 청장년 및 노인(Mackersie et al., 2015) 이 포함되었다.

측정 방법에 따른 분류에 있어서 객관적 측정을 수행한 14 건 의 문헌 중에는 동공 확장(Alhanbali et al., 2019; Ayasse et al., 2017; Ayasse \& Wingfield, 2018) 및 안구 움직임의 정확도 (Tun et al., 2009), EEG (Alhanbali et al., 2019) 및 사건 유발 전위(event-related potential, ERP)에 포함되는 P50-N1-P2 complex (Giroud et al., 2017), N1반응(Bernarding et al., 2013), P3반응(Bertoli \& Bodmer, 2014), 피부 전도성(Alhanbali et al., 2019; Mackersie et al., 2015), 호흡수 및 심박수 (Mackersie et al., 2015)가 포함되었다. 한편, 주관적 측정을 수 행한 11건의 문헌 중에는 정확도(Desjardins \& Doherty, 2013; Ward et al., 2017, Picou \& Ricketts, 2018; Ricketts et al., 2019), 반응 시간(Alhanbali et al., 2019; Ricketss et al., 2019; Ward et al., 2017), 응답 시간(Picou \& Ricketts, 2018) 및 작업 기억의 능력을 평가하는 회상 정확도(Tun et al., 2009), 종합 기호 잇기 검사(Rosemann \& Thiel, 2020), 단어 회상 정확도 (Bertoli \& Bodmer, 2014)가 포함되었다. 마지막으로 자가 보 고 측정을 수행한 10 건의 문헌들은 모두 척도에 기반한 설문 문항을 사용하였으며, 10점 척도(Alhanbali et al., 2019; Rosemann \& Thiel, 2019; Rosemann \& Thiel, 2020; Zekveld et al., 2009)가 가장 많이 적용되었다. 이어서 7점 척도(Holube et al., 2016; Huber et al., 2018; Kruger at al., 2017), 11점 척도 (Ricketts et al., 2019), 20점 척도(Alhanbali et al., 2019), 100 점 척도(Desjardins \& Doherty, 2013)의 순으로 다양한 척도가 사용되었다.

\section{Overall effectiveness of measurements for listening effort} 변량 효과 모형을 사용한 전체 문헌의 효과 크기 결과는 Figure $2 \mathrm{~A}$ 에 제시하였다. 전체 문헌의 표준화된 평균 차이는 1.031(95\% CI: -0.106 2.169)을 보였다. 청취 노력의 측정 방법 에 따른 효과를 확인하기 위해 세가지 측정 방법에 따른 하위 군 분석을 수행하였으며, 객관적 측정에 따른 청취 노력의 결과 는 1.686의 효과 크기와 통계적 유의미성으로(95\% CI: 1.233 


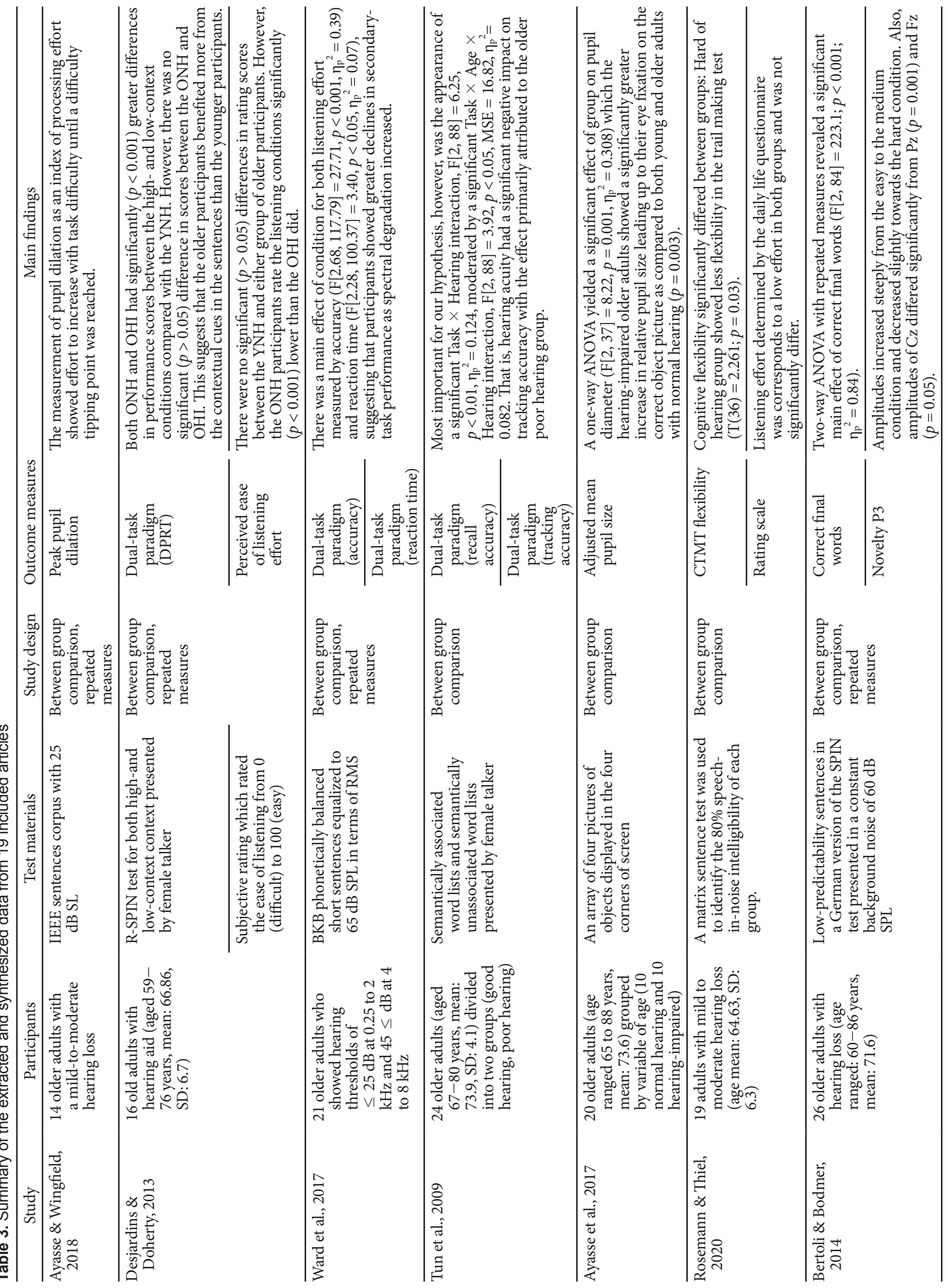




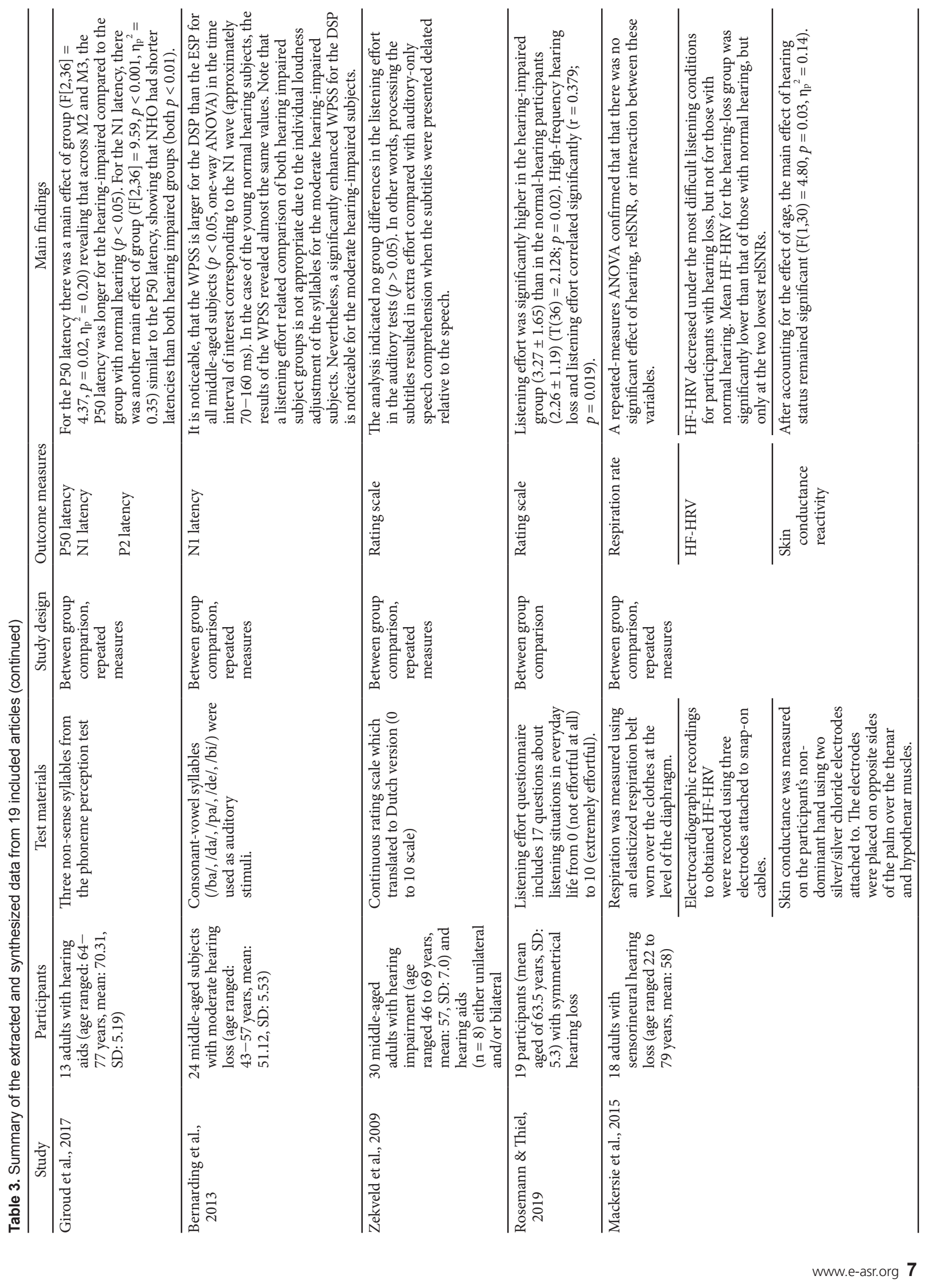




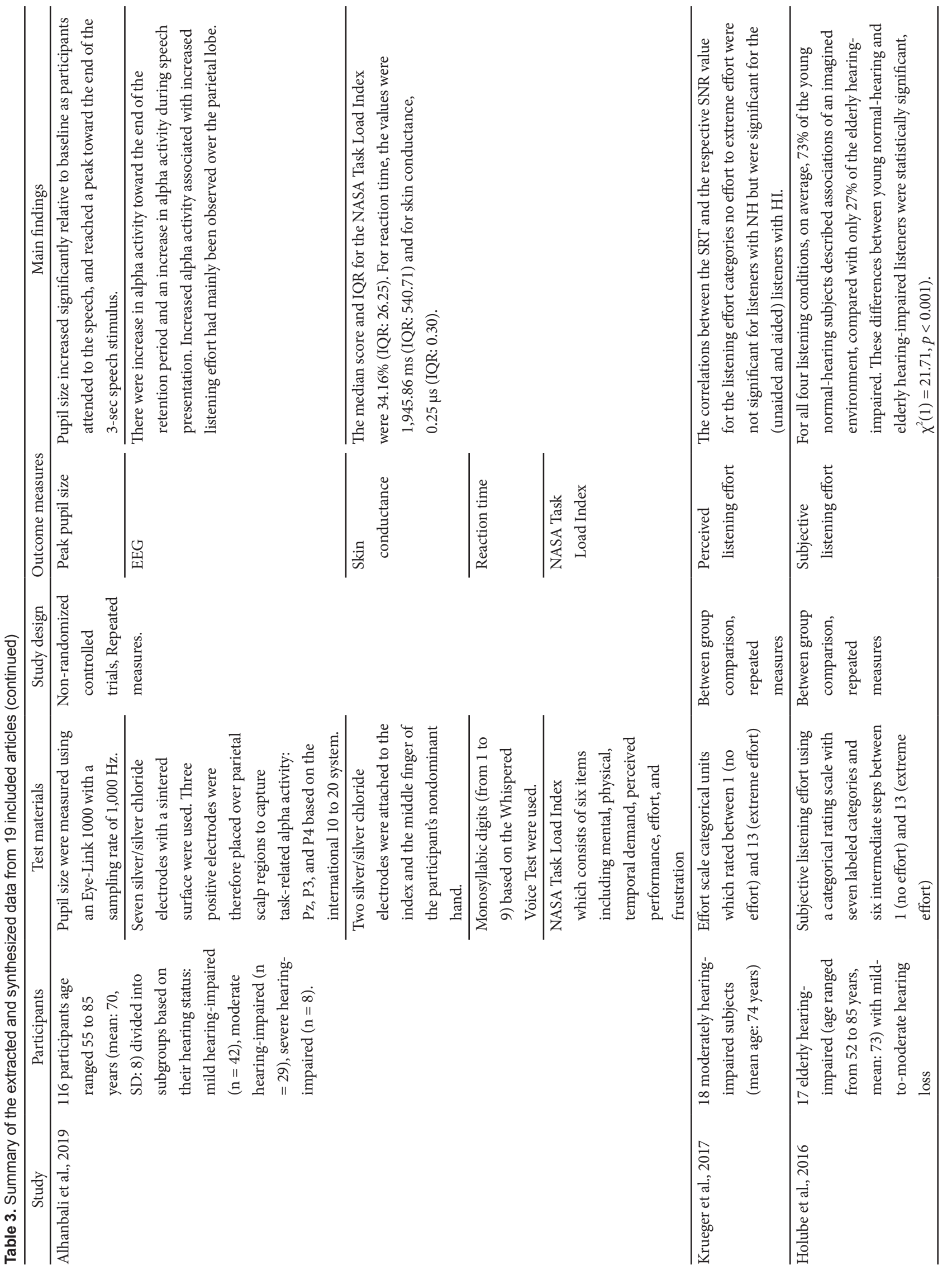




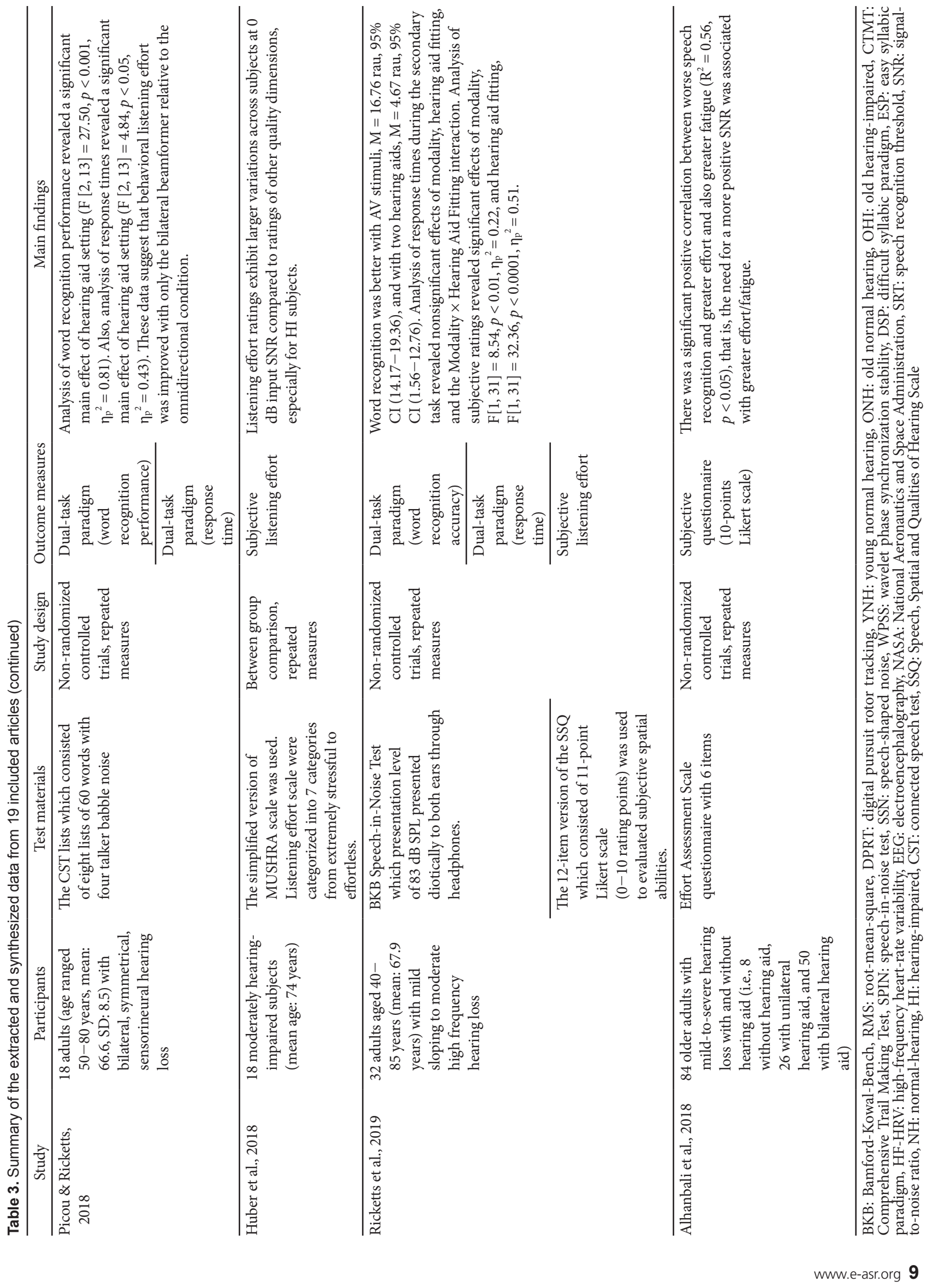


$2.490, p=0.001)$ 가장 높은 효과 크기를 보였다. 자가 보고 측 정과 주관적 측정에 따른 청취 노력의 결과 역시 각각 -1.280 (95\% CI: -4.180 1.620, $p=0.387$ )와 1.221 (95\% CI: 0.267
$2.175, p=0.012)$ 의 효과 크기를 보였으며 자가 보고 측정을 제 외한 모든 측정 방법에서 통계적 유의미성이 검정되었다. 하위 군별 결과를 보다 자세히 확인하고자 결과 측정 방법에 따른

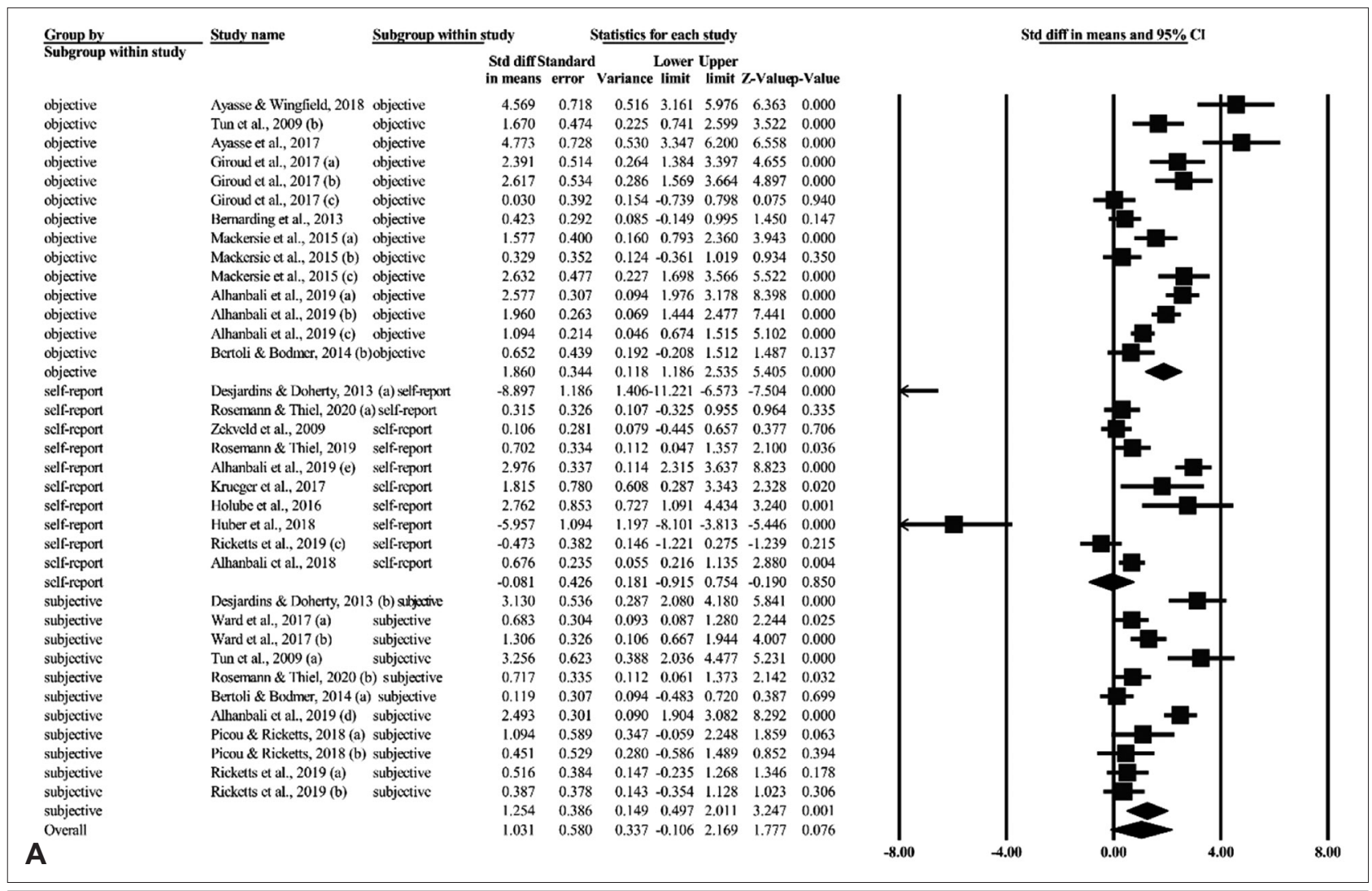

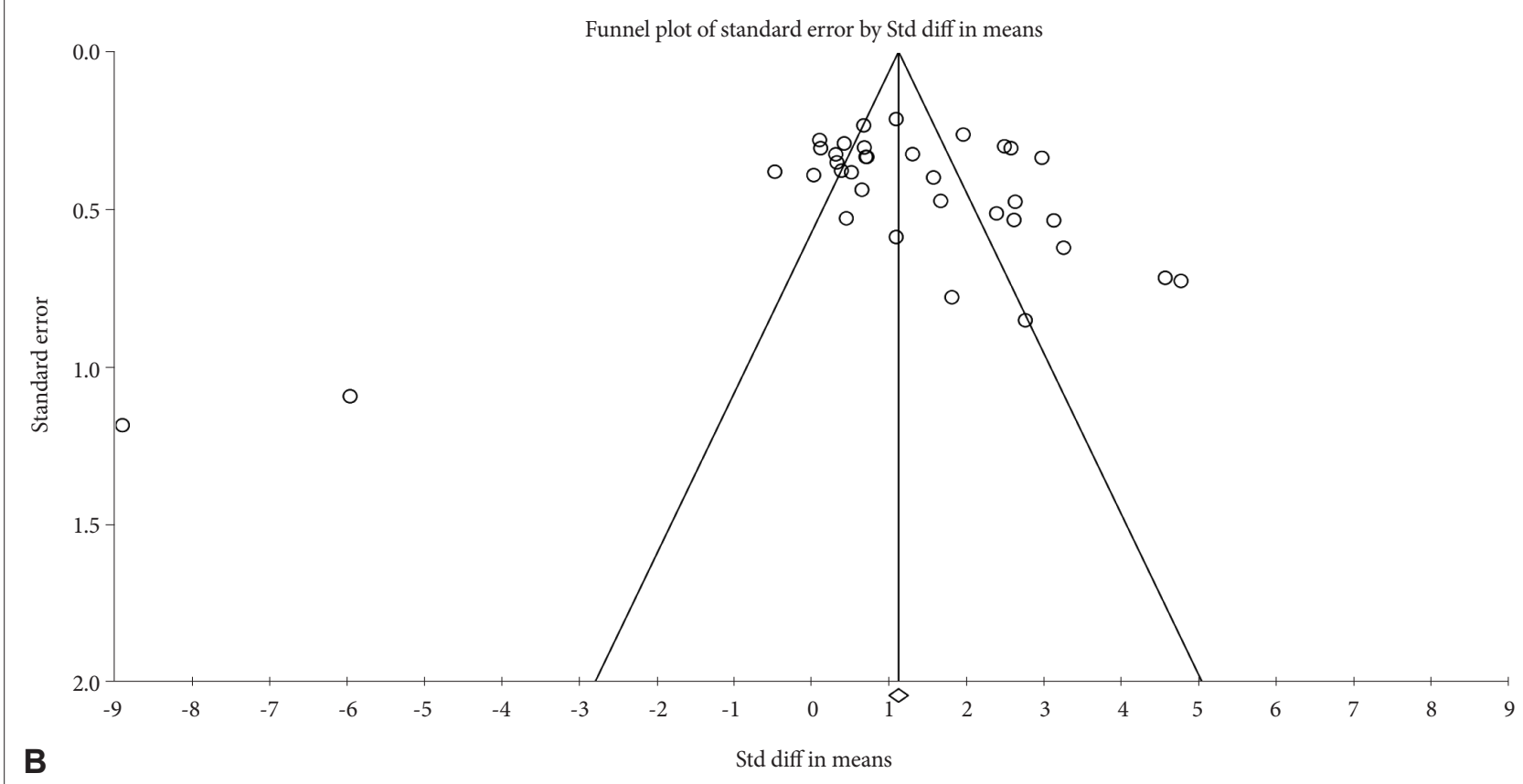

Figure 2. Forest plot (A) which showed effect size based on standardized difference in means, and funnel plot (B) which presented an asymmetrical shape of an all included 35 studies. Cl: confidence interval. 
효과를 추가적으로 분석하였다.

Figure 2B에 제시한 깔때기 그림은 좌측으로 치우치는 편측 성을 보였으나, Egger's regression asymmetry test 결과 출판 편향이 없음을 확인하였다(intercept: 0.29186, standard error: $1.68084, p=0.43160$ ). 전체 문헌에 대한 이질성은 Cochrane's $\mathrm{Q}$ test에서 유의미함을 보였으며(Q: 385.990, df: 34, $p$ < 0.001), Higgin's $I^{2}$ test 결과 역시 높은 수준의 이질성을 보였기 때문 에 $\left(\mathrm{I}^{2}:\right.$ 91.191), 사전에 설정한 하위군 분석을 통해 청취 노력의 측정 방법(객관적 측정, 자가 보고 측정, 주관적 측정)에 따른 비교를 수행하였다.

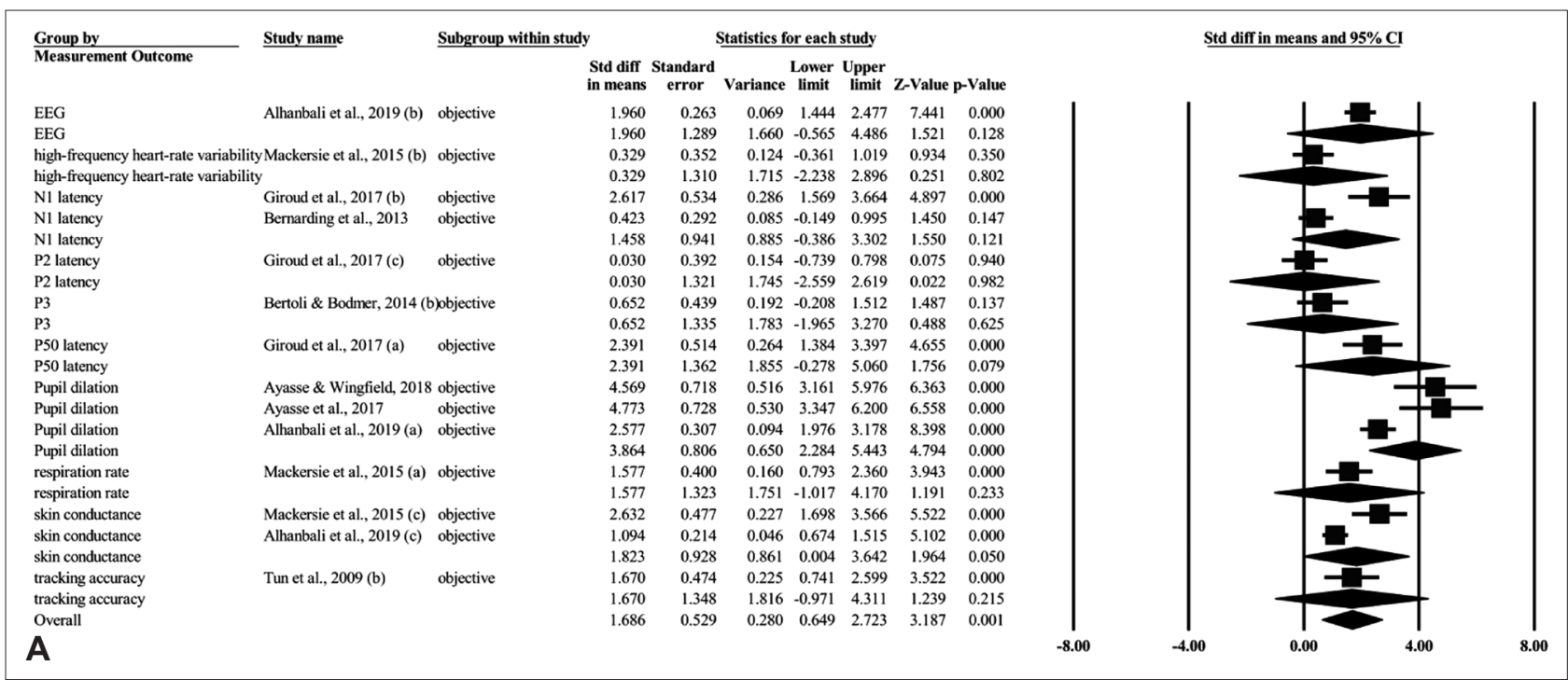

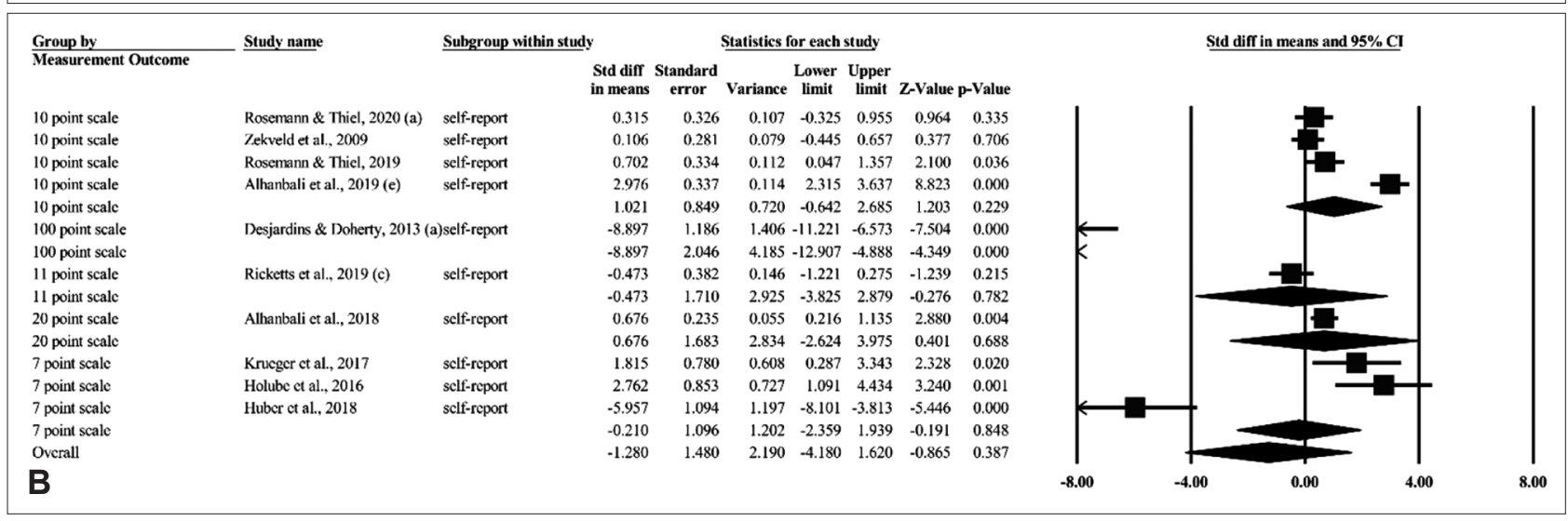

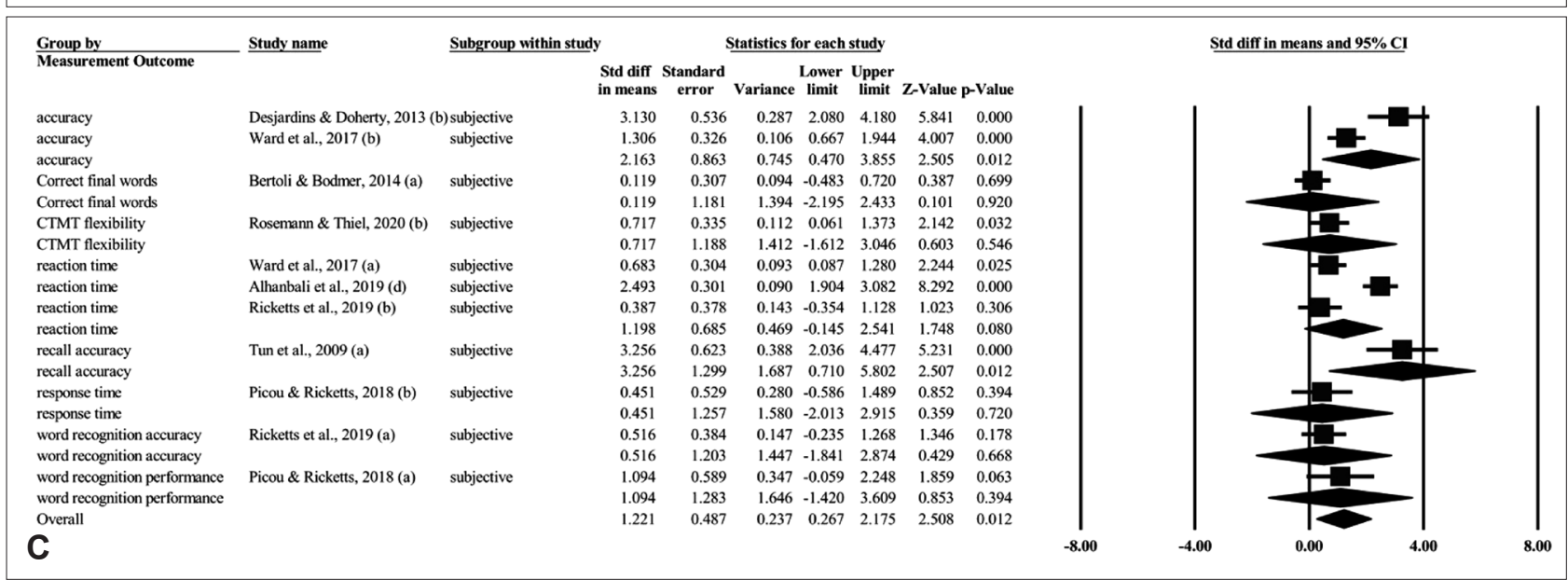

Figure 3. Results of subgroup analysis based on the measurement type of listening effort. Objective measurement (A), self-report measurement (B), subjective measurement (C). EEG: electroencephalography, CTMT: Comprehensive Trail-Making Test, Cl: confidence interval. 


\section{Effect of outcome measurement on listening effort}

Figure $3 \mathrm{~A}$ 에 제시된 객관적 측정 방법에 따른 효과를 확인했 을 때, 결과 측정 방법으로 동공 확장(pupil dilation)을 사용하 는 것이 가장 큰 효과 크기와 통계적 유의미성을 나타냈다(effect size: 3.864, 95\% CI: 2.284 5.443, $p<0.001$ ).

자가 보고 측정의 결과, 측정 방법에 따른 효과는 Figure $3 \mathrm{~B}$ 에 제시되었다. 사용된 척도별 효과를 확인했을 때, 10 점 척도 를 사용하는 것이 1.021 로 가장 큰 효과 크기를 보였으나, 통계 적 유의미성은 확인되지 않았다 $(95 \%$ CI: $-0.642 ~ 2.685, p=$ 0.229). 반면에, 100 점 척도를 사용한 결과는 통계적 유의미성 은 확인되었으나, 가장 낮은 효과 크기를 보였다(effect size: -8.897, 95\% CI: -12.907 4.888, $p<0.001$ ).

Figure 3 C에 제시된 주관적 측정의 결과 측정 방법에 따른 효과는 단어 회상(word recall)을 활용한 정확도(effect size: 3.256, 95\% CI: 0.710 5.802, $p=0.012$ )와 정확도(effect size: 2.163, 95\% CI: 0.470 3.855, $p=0.012$ )를 사용하는 것이 큰 효 과 크기를 보였다.

\section{CONCLUSIONS}

본 논문에서는 난청 성인 및 보청기 착용 성인의 청취 노력 측정에 사용되는 측정 방법의 정확성과 효율성을 확인하기 위 해 체계적 문헌 고찰 및 메타 분석을 수행하였다. 난청 성인 및 보청기 착용 성인의 청취 노력에 대해 측정한 총 19 편의 개별 문헌의 데이터를 추출 및 합성하여, 총 35 건의 데이터를 측정 방법에 따라 세개의 하위 그룹으로 분류한 후 메타 분석을 수 행하였다. 객관적 측정과 주관적 측정은 난청 및 보청기 착용 성인의 청취 노력을 측정함에 있어서 효과가 확인되었으나, 아 쉽게도 청취 노력의 측정에 있어서 자가 보고 측정의 효과는 입증되지 않았다. Pichora-Fuller et al.(2016)의 연구에서는 청 취 노력의 주관적 측정 결과와 자가 보고 측정 결과의 불일치 성에 대해 보고한 바 있다. 즉, 청취 노력은 동기 부여, 인지적 자원, 노력의 3차원적인 인지 과정의 산출물이기 때문에 수많 은 인지적 처리 과정에 영향을 받게 된다. 구체적으로 주관적 측정 결과와 자가 보고 결과의 불일치성은 개인별 각성 상태에 영향을 받으며, 이는 동기부여에도 부정적인 영향을 미치게 된 다. 높은 각성 상태에서는 어려운 청취 환경 및 과제에 대해 높 은 동기부여를 기대할 수 있으며 이는 높은 인지적 자원의 사 용과 노력을 통해 높은 청취 노력의 발현을 보이게 되지만, 낮 은 각성 상태에서는 동일한 환경 및 과제에 대해 저조한 동기부 여로 인해 인지적 자원의 사용이 낮아지게 되고 이는 낮은 청 취 노력을 야기하게 된다.

청취 노력의 측정에 대해 가장 큰 효과를 보인 객관적 측정
방법에서는 동공 확장을 사용하여 청취 노력을 측정하는 것이 가장 효율적인 것으로 나타났으며, 주관적 측정 방법과 자가 보고 측정 방법에서는 각각 작업 기억을 활용한 회상 정확도와 10 점 척도를 사용하는 것이 효율적인 것으로 확인되었다. 그러 나 가장 큰 효과를 보였던 객관적 측정 방법뿐만 아니라 주관 적 측정과 자가 보고 측정 방법의 효과는 결과 측정 방법에 따 라서 청취 노력의 측정에 대한 효과에 상당한 차이를 보였다. 객관적, 주관적, 자가 보고 측정 방법에 따른 청취 노력의 결과 간 상관성을 비교 분석한 Alhanbali et al.(2019)의 연구에서는 청취 노력의 측정 방법과 환경에 따라 청취 노력이 다르게 나타 날 수 있으며 이는 청취 노력이 다차원적 개념이기 때문에 발생 가능하다고 보고하였다. 이는 Kahneman (1973)의 인지 능력 모델과 Pichora-Fuller et al.(2016)의 FUEL에서의 보고와 동 일하게 청취(listening)는 제시되는 소리를 청각 기관을 사용하 여 듣고 정보를 분석하여 뇌로 전달하게 되며 뇌로 전달된 정 보는 지각 및 이해의 과정을 거쳐 기억(memory)의 형태로 저 장되는 청각-인지적 상호작용의 과정으로 해석할 수 있다. 또 한, 청취로 인해 유발되는 청각-인지적 상호작용 중 인지적 기 능의 관점에서는 작업 기억, 집중력, 처리 속도 등이 포함되게 되며 궁극적으로는 인지적 자원의 소모와 이로 인한 청취 노력 을 야기시킨다(Alhanbali et al., 2019; Pichora-Fuller et al., 2016). 다시 정리해보면, 다차원적 개념인 청취 노력을 측정하기 위해 현재까지 동공 확장이나 $\mathrm{EEG}$ 등의 객관적 측정, 정확도 와 반응 시간을 포함하는 주관적 측정, 대상자의 주관적인 응 답에 의한 자가 보고 측정의 방법 등이 사용되고 있으나, 각 측 정 방법의 개별적인 측정 결과는 청취 노력의 단편적인 정보만 을 제공하기 때문에(Alhanbali et al., 2018; Alhanbali et al., 2019; Ayasse et al., 2017; Ayasse \& Wingfield, 2018; Pichora-Fuller et al., 2016) 보다 종합적인 측정 과정과 이에 대한 분석이 필요할 것으로 사료된다.

임상적 관점에서 난청인과 보청기 사용자의 청취 노력의 측 정 방법에 대한 적용은 측정 방법에 따른 효과와 현실적인 환 경에 따라 구분될 수 있다. 예를 들어, 가장 우수한 검사법으로 분석된 동공 확장이나 $\mathrm{EEG}$ 와 같은 객관적 측정 방법은 특수 장비 및 시설을 구비한 상급병원 등의 임상 기관에서 적용이 가능하거나, 주관적 측정 방법인 작업 기억 검사 및 해석은 타 분야의 전문가와 협력이 가능할 때 보다 효과적이고 효율적으 로 난청인 및 보청기 사용자의 청취 노력을 정량적으로 측정할 수 있을 것으로 사료된다. 반면, 음장 검사를 활용하여 다양한 청취 환경에서 어음인지의 정확도 및 반응/응답 시간의 측정하 는 주관적 측정 방법은 클리닉 및 청각 센터에서의 적용 가능 하겠다. 또한, 난청인 및 보청기 사용자가 주관적으로 느끼는 지수를 정량적으로 평가하기 위한 자가 보고 측정법을 객관적, 
주관적 측정법과 함께 사용하여 검사대상자의 중심의 주관적

결과를 보완하는 것 또한 매우 바람직하다.

결론적으로, 본 논문은 난청인과 보청기 착용자에게 요구되 는 청취 노력의 측정 방법에 따른 효과를 체계적 문헌 고찰과 메타 분석에 근거하여 작성했다는데 의의를 둘 수 있다. 향후에 는 높은 근거 기반 연구(evidence-based research)등 질적 우 수성이 확보된 연구를 통해 난청인과 보청기 착용자의 청취 노 력 측정에 대한 효율성 확인과 임상적 검증이 필요할 것이다. 더불어 난청인 및 보청기 사용자를 대상으로 어음인지도와 청 취 노력 간 상관성을 정확하게 분석하고 이를 근거로 객관적 지 표를 제시한다면 청취 노력의 과학적 측정 근거가 될 것이며 대 상자의 어음인지도 평가, 보청기 적합, 인지적 자원의 사용에 대한 상담 등에 청취 노력의 임상적 활용도가 높을 것으로 생 각된다.

중심 단어 : 보청기·난청·청취 노력·객관적 측정·자가보고· 주관적 측정.

\section{Ethical Statement}

N/A

\section{Acknowledgments \\ N/A}

\section{Declaration of Conflicting Interests}

There are no conflict of interests.

\section{Funding}

This work was supported by the National Research Foundation of Korea (NRF) grant funded by the Korea government (MSIT) (2019R1F1A 1053060).

\section{Author Contributions}

Conceptualization: all authors. Formal analysis: Chanbeom Kwak. Funding acquisition: Woojae Han. Methodology: all authors. Supervision: Woojae Han. Validation: Woojae Han. Visualization: Chanbeom Kwak. Writing_original draft: Chanbeom Kwak. Writing_review \& editing: Woojae Han. Approval of final manuscript: all authors.

\section{ORCID iDs}

Chanbeom Kwak https://orcid.org/0000-0001-5657-7536

Woojae Han https://orcid.org/0000-0003-1623-9676

\section{REFERENCES}

Alhanbali, S., Dawes, P., Lloyd, S., \& Munro, K. J. (2018). Hearing handicap and speech recognition correlate with self-reported listening effort and fatigue. Ear and Hearing, 39(3), 470-474.

Alhanbali, S., Dawes, P., Millman, R. E., \& Munro, K. J. (2019). Measures of listening effort are multidimensional. Ear and Hearing, 40(5), 10841097.

Ayasse, N. D., Lash, A., \& Wingfield, A. (2017). Effort not speed characterizes comprehension of spoken sentences by older adults with mild hearing impairment. Frontiers in Aging Neuroscience, 8, 329.

Ayasse, N. D. \& Wingfield, A. (2018). A tipping point in listening effort: Effects of linguistic complexity and age-related hearing loss on sentence comprehension. Trends in Hearing, 22, 2331216518790907.

Bernarding, C., Strauss, D. J., Hannemann, R., Seidler, H., \& CoronaStrauss, F. I. (2013). Neural correlates of listening effort related factors: Influence of age and hearing impairment. Brain Research Bulletin, 91, 21-30.

Bertoli, S. \& Bodmer, D. (2014). Novel sounds as a psychophysiological measure of listening effort in older listeners with and without hearing loss. Clinical Neurophysiology, 125(5), 1030-1041.

Centre for Reviews and Dissemination. (2009). Systematic Reviews: CRD's Guidance for Understanding Reviews in Health Care. York University, Centre for Reviews and Dissemination. Retrieved from https://www. york.ac.uk/media/crd/Systematic_Reviews.pdf.

Desjardins, J. L. \& Doherty, K. A. (2013). Age-related changes in listening effort for various types of masker noises. Ear and Hearing, 34(3), 261272.

Giroud, N., Lemke, U., Reich, P., Matthes, K. L., \& Meyer, M. (2017). The impact of hearing aids and age-related hearing loss on auditory plasticity across three months - An electrical neuroimaging study. Hearing Research, 353, 162-175.

GRADE Working Group. (2004). Grading quality of evidence and strength of recommendations. BMJ, 328(7454), 1490.

Higgins, J. P., Thompson, S. G., Deeks, J. J., \& Altman, D. G. (2003). Measuring inconsistency in meta-analyses. BMJ, 327(7414), 557-560.

Holube, I., Haeder, K., Imbery, C., \& Weber, R. (2016). Subjective listening effort and electrodermal activity in listening situations with reverberation and noise. Trends in Hearing, 20, 2331216516667734.

Huber, R., Bisitz, T., Gerkmann, T., Kiessling, J., Meister, H., \& Kollmeier, B. (2018). Comparison of single-microphone noise reduction schemes: can hearing impaired listeners tell the difference? International Journal of Audiology, 57(sup3), S55-S61.

Kahneman, D. (1973). Attention and Effort. (1st ed.). Englewood Cliffs, NJ: Prentice-Hall.

Kang, H. (2015). Statistical considerations in meta-analysis. Hanyang Medical Reviews, 35(1), 23-32.

Krueger, M., Schulte, M., Zokoll, M. A., Wagener, K. C., Meis, M., Brand, T., et al. (2017). Relation between listening effort and speech intelligibility in noise. American Journal of Audiology, 26(3S), 378-392.

Mackersie, C. L., MacPhee, I. X., \& Heldt, E. W. (2015). Effects of hearing loss on heart rate variability and skin conductance measured during sentence recognition in noise. Ear and Hearing, 36(1), 145-154.

McGarrigle, R., Munro, K. J., Dawes, P., Stewart, A. J., Moore, D. R., Barry, J. G., et al. (2014). Listening effort and fatigue: What exactly are we measuring? A British Society of Audiology Cognition in Hearing Special Interest Group 'white paper'. International Journal of Audiology, 53(7), 433-440

Moher, D., Shamseer, L., Clarke, M., Ghersi, D., Liberati, A., Petticrew, M., et al. (2015). Preferred reporting items for systematic review and metaanalysis protocols (PRISMA-P) 2015 statement. Systematic Reviews, $4(1), 1$.

Pichora-Fuller, M. K., Kramer, S. E., Eckert, M. A., Edwards, B., Hornsby, B. W., Humes, L. E., et al. (2016). Hearing impairment and cognitive energy: The Framework for Understanding Effortful Listening (FUEL). Ear and Hearing, 37 Suppl 1, 5S-27S.

Picou, E. M. \& Ricketts, T. A. (2018). The relationship between speech recognition, behavioural listening effort, and subjective ratings. International Journal of Audiology, 57(6), 457-467.

PROSPERO. (2014). International Prospective Register of Systematic Reviews. York University, Centre for Reviews and Dissemination. Retrieved from http://cdn.elsevier.com/promis_misc/PROSPEROAnimal.pdf.

R Core Team. (2018). R: A Language and Environment for Statistical Computing [Software]. Vienna, Austria: R Foundation for Statistical Computing. Retrieved from: http://www.R-project.org/.

Ricketts, T. A., Picou, E. M., Shehorn, J., \& Dittberner, A. B. (2019). Degree of hearing loss affects bilateral hearing aid benefits in ecologically relevant laboratory conditions. Journal of Speech, Language, and Hearing 
Research, 62(10), 3834-3850.

Rosemann, S. \& Thiel, C. M. (2019). The effect of age-related hearing loss and listening effort on resting state connectivity. Scientific Reports, 9(1), 2337.

Rosemann, S. \& Thiel, C. M. (2020). Neural signatures of working memory in age-related hearing loss. Neuroscience, 429, 134-142.

Tun, P. A., McCoy, S., \& Wingfield, A. (2009). Aging, hearing acuity, and the attentional costs of effortful listening. Psychology and Aging, 24(3), 761-766.

Ward, K. M., Shen, J., Souza, P. E., \& Grieco-Calub, T. M. (2017). Age-re- lated differences in listening effort during degraded speech recognition. Ear and Hearing, 38(1), 74-84.

Wingfield, A., McCoy, S. L., Peelle, J. E., Tun, P. A., \& Cox, L. C. (2006). Effects of adult aging and hearing loss on comprehension of rapid speech varying in syntactic complexity. Journal of the American Academy of Audiology, 17(7), 487-497.

Zekveld, A. A., Kramer, S. E., Kessens, J. M., Vlaming, M. S., \& Houtgast, T. (2009). The influence of age, hearing, and working memory on the speech comprehension benefit derived from an automatic speech recognition system. Ear and Hearing, 30(2), 262-272. 